\title{
CHEMICAL GRAPHS-V
}

\section{ENUMERATION AND PROPOSED NOMENCLATURE OF BENZENOID CATA-CONDENSED POLYCYCLIC AROMATIC HYDROCARBONS ${ }^{2}$}

\author{
A. T. BALABAN and F. HARARY ${ }^{2}$ \\ Institute of Atomic Physkcs, Bucharest, Roumanis: University of Michigan, Ann Arbor, U.S.A.: \\ and University College. London
}

(Recerved in the UK is July 1967; accepted for publication 14 September 1967)

\begin{abstract}
Alutact - A modified definition is proposod for cata-condeasod aromatic hydrocarbons. A formula is devised for the number of isomers of non-braxched cata-condensed arenes in function of the number of hexagons. Proposals are made for a graph-systematic nomenclature of such arenes, based on the characteristic tree of these systems.
\end{abstract}

THE nomenclature of benzenoid condensed polycyclic aromatic hydrocarbon is extremely complex. In Clar's two-volume monograph ${ }^{3}$ the summary extends over $13+48$ pages, since the German nomenclature system adopted therein is different from the IUPAC, ${ }^{4}$ Chemical Abstracts ${ }^{5}$ or Ring Index ${ }^{6}$ systems, and it was necessary to depict the formulae of all compounds discussed in order to indicate rapidly and unambiguously which compounds were discussed. This fact illustrates the basic faults of all nomenclature systems adopted so far in the class of benzenoid polycyclic hydrocarbons. Trivial names (coronene, pyrene, ovalene, violanthrene, zethrene, chrysene, picene, circumanthracene, perylene, terrylene, anthanthrene, bisanthene. peropyrene, (iso)violanthrene, pyranthrene, etc.) along with semisystematic names (acenes, phenes, helicenes, rylenes) and with different alternative systems for indicating by numbers ${ }^{3}$ or letters ${ }^{-6}$ the homology (benzo, naphtho, etc.) make the nomenclature extremely confusing.

No attempt has apparently been made to devise a formula for counting the various isomeric polycyclic aromatic hydrocarbons. In the present paper we indicate a counting series for a part of the polycyclic hydrocarbons, proposing at the same time a simple nomenclature system. This may be useful for computer handling of literature data (cf.'), taking into account that many systems falling under the proposed nomenclature have important applications in cancer research," in the dyestuff industry, and in theoretical, physical and organic chemistry.3.10

All benzenoid polycyclic aromatic hydrocarbons (arenes) are superimposable with the graphite lattice. They are classified into several groups as shown in Fig. 1.* Condensed (fused $\dagger$ ) polycyclic arenes have two adjacent carbon atoms common to two rings. like naphthalene $I$.

- In formulas 1-XV. double bonds or inscribed circles in aromatic rings have ben omitted for clarity.

in Ine IUPAC 1957 Rules, cato-condensed systems are named ortho-fused and pert-condensed systems are named ortho- and perifused. 


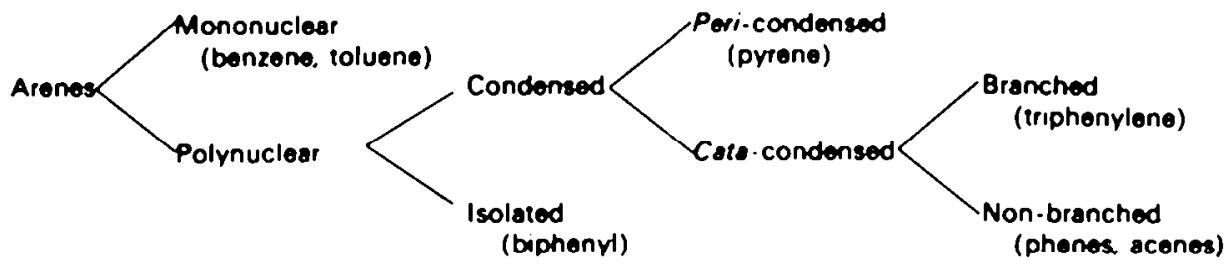

Fig. I ('lassification of arenes (examples are given in brackets)

The usual criterion for classifying a condensed polycyclic arene as cata- or pericondensed is the absence or presence of a $\mathrm{C}$ atom common to three rings. We propose a modified, graph-theoretical definition of cata- or peri-condensed systems. If the centers of the hexagons are joined by a line when two hexagons are condensed, one obtains a connected graph which will be named "characteristic graph" or "skeleton". By the new, proposed, definition if this graph is a tree, the arene is cata-condensed; if this graph is not a tree (if it contains rings), the arene is peri-condensed.<smiles>CC12CCCCC1CCCC2</smiles>

I<smiles>CC1CCCC2CCCCC1C21CCCCC2CCCCC21</smiles>

II

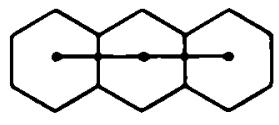

III

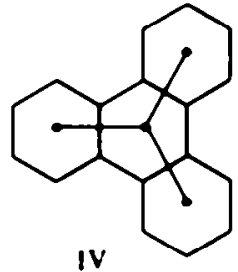

IV

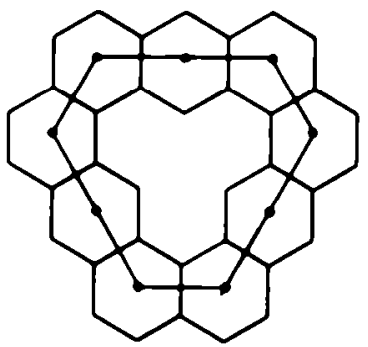

v

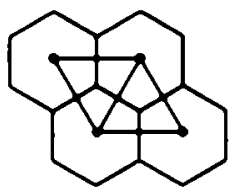

VII

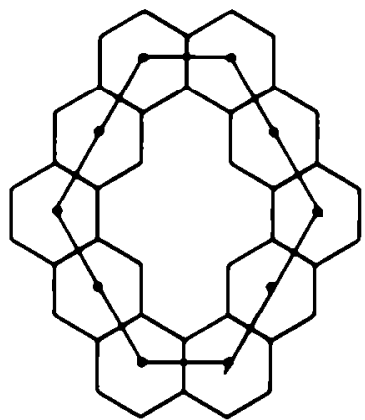

VIA

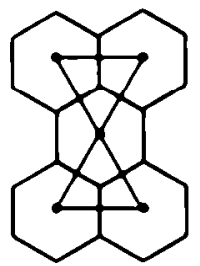

VIII

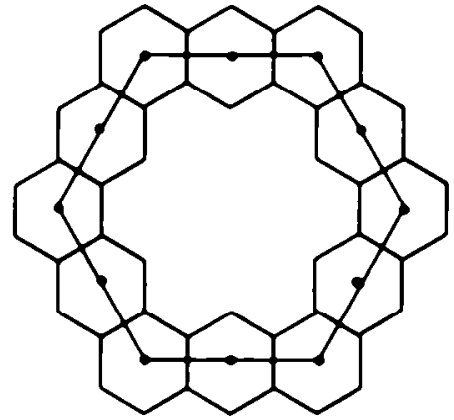

VIB

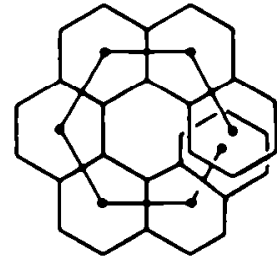

IX 
Systems I-IV are classified as cata-condensed both by the usual and by the new definition; likewise, pyrene VII and perylene VIII are peri-condensed systems in both definitions. The two definitions are, however, at variance in the case of systems such as V, VIA ${ }^{110}$ and VIB, ${ }^{110}$ which are cata-condensed in the usual definition, but peri-condensed in the new one. We favour the new definition because if this is accepted, all cata-condensed arenes with the same number $n$ of hexagons are isomeric, i.e. have the same molecular formula $\mathrm{C}_{4 n+2} \mathrm{H}_{2 n+4}$. This is not true for peri-condensed systems, both in the usual and in the new definitions.

Compound $\mathrm{V}, \mathrm{C}_{36} \mathrm{H}_{18}$ is not an isomer of nonacene or of other cata-condensed hydrocarbons with nine hexagons $\mathrm{C}_{38} \mathrm{H}_{22}$, neither are VIA, $\mathrm{C}_{40} \mathrm{H}_{16}$, and VIB, $\mathrm{C}_{48} \mathrm{H}_{24}$, isomers of decacene, $\mathrm{C}_{42} \mathrm{H}_{24}$ and dodecacene, $\mathrm{C}_{30} \mathrm{H}_{28}$, respectively; similarly the peri-condensed system pyrene VII, $\mathrm{C}_{16} \mathrm{H}_{10}$ is not an isomer of tetracene $\mathrm{C}_{18} \mathrm{H}_{12}$, neither is perylene VII, $\mathrm{C}_{20} \mathrm{H}_{12}$, an isomer of pentacene $\mathrm{C}_{22} \mathrm{H}_{14}$. Accordingly, it is reasonable to classify V, VIA, VIB and other coronaphenes as peri-condensed systems."

Compounds like hexahelicene or the recently prepared ${ }^{12}$ heptahelicene IX which are non-planar and cannot be drawn on paper (therefore they are not strictly superposable with the graphite lattice) can be considered as cata-condensed systems.

By adopting henceforth the new definition of cata- and peri-condensed systems, we may hope to be able to devise a formula giving the number $C_{\text {. }}$ of the isomers of a given cata-condensed system with $n$ hexagons. The solution of this problem will be presented in this paper together with a new nomenclature system of cata-condensed arenes.

This also provides a partial answer to the hexagonal cell-growth problem, which was repeatedly mentioned by one of us ${ }^{13-16}$ as one of the unsolved problems in the theory of graphs. This problem, which can be formulated: "how many hexagonal-cell aggregates or "animals" are there for a given number $n$ of hexagons ", is equivalent to counting all condensed (cat $a$ and peri) arenes with $n$ hexagons.

By empirical approaches the condensed polycyclic arenes shown in Fig. 2 and presented in Table 1 have been found.

The peri-condensed systems present many complicating features: when their characteristic graph has an odd number of triangles, they are radicals ( $r$ in Table 1) like perinaphthyl $X$, or naphtanthryl $X I$; with an even number of triangles, they can be diradicals (d) like coeranthrene XII, or like XIII and XIV, or can be "normal" (p) like pyrene VII and perylene VIII. In the former case, it is impossible to accommodate one double bond at every carbon atom in the sense of Kekule structures. If all atoms are distributed into two alternate sets of starred and non-starred atoms, ${ }^{17}$ the sets

\footnotetext{
- Altematively, one could conserve the previous meaning of peri-condensed systems by ascribing this name to compounds whose skeleton contains three-membered rings (trangles), and by coining for com. pounds such as $V$ or VI, whose skeleton has a larger ring, a new name, $e . g$ corona-condensed systems: besides other interesting features such as ring curfent or clathrate formation these latter systems could kad to Möbius-strip molecules, if two adjacent bonds change places
}

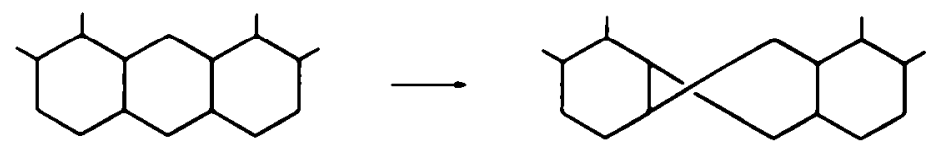




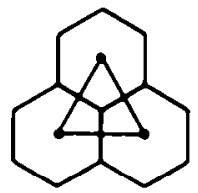

$\mathbf{x}$

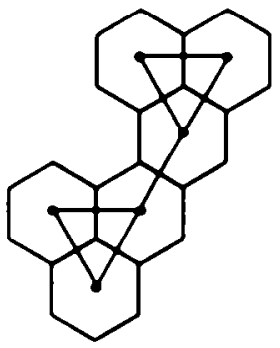

XIII

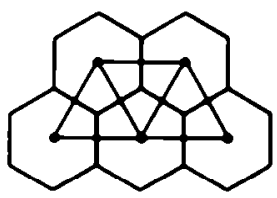

XI

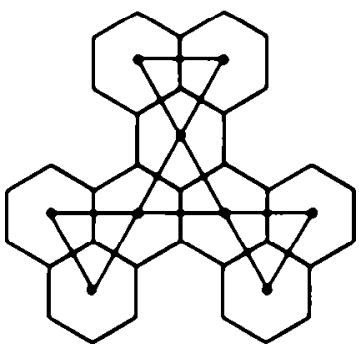

XIV

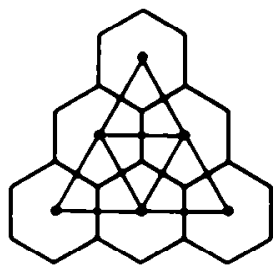

$\mathbf{X I I}$

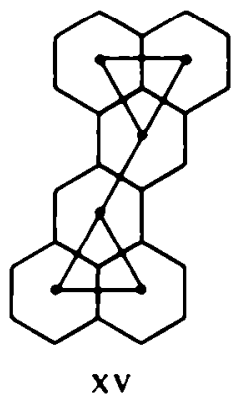

have equal numbers of atoms in normal compounds and unequal in diradicals." Structural features (z) like those present in zethrene XV determine the localization of the double bonds shown in the formula. Since the Hückel rule of aromaticity breaks down in peri-condensed aromatic systems it is mostly in cata-condensed compounds that Kekule structures are important, as evidenced by Clar ${ }^{18}$ or by correlations $^{19}$ between the maximum number of rings in which three alternate double bonds can be written and the delocalization energy.

Starting from the observation ${ }^{20}$ that in the graphite lattice all lines have one of three directions which can be noted by digits $\pm 1, \pm 2, \pm 3$ similarly to the fourdirection problem in the diamond lattice, ${ }^{21}$ one can describe the outline of a condensed arene by a number consisting of these digits, e.g. benzene by $132 T 32$ and
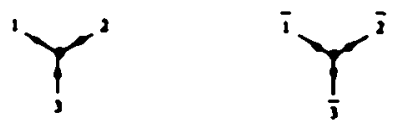<smiles>C1=CCCCCC1</smiles>

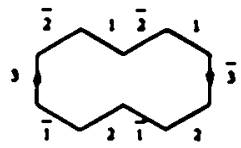

HI. 3

naphthalene by $132 T 2 T 3212$ (Fig. 3). This approach seems suitable for computer use. These numbers must have several restrictions: every positive digit must have in the number its negative counterpar; two adjacent digits must have opposite signs; only sequences, 12, 13, 2I, 23, 3I, 32 and the same with reversed signs, are allowed.

A simpler approach is to consider the characteristic graph associated to the arene. Owing to the complications introduced by peri-condensed systems, we shall discuss in the following only cata-condensed compounds. Their graph is a tree, which may be branched or non-branched; in the latter case the tree has only two endpoints.

- Triangular artangements of hexagons like X. XII, etc., kead to monoradicals diradicals. .., q-radicals. Such \& q-radical is a triangular array of $\mathrm{H}(q+1 \mathrm{Xq}+2)$ hexagons (one side of the equilateral triangle consists of $q+1$ hexagons), containing $H(q+6)(q+1)$ starred and $H(q+3)(q+2)$ non-starrod atoms. 
fk: 2 Condensed polyoptix arenes

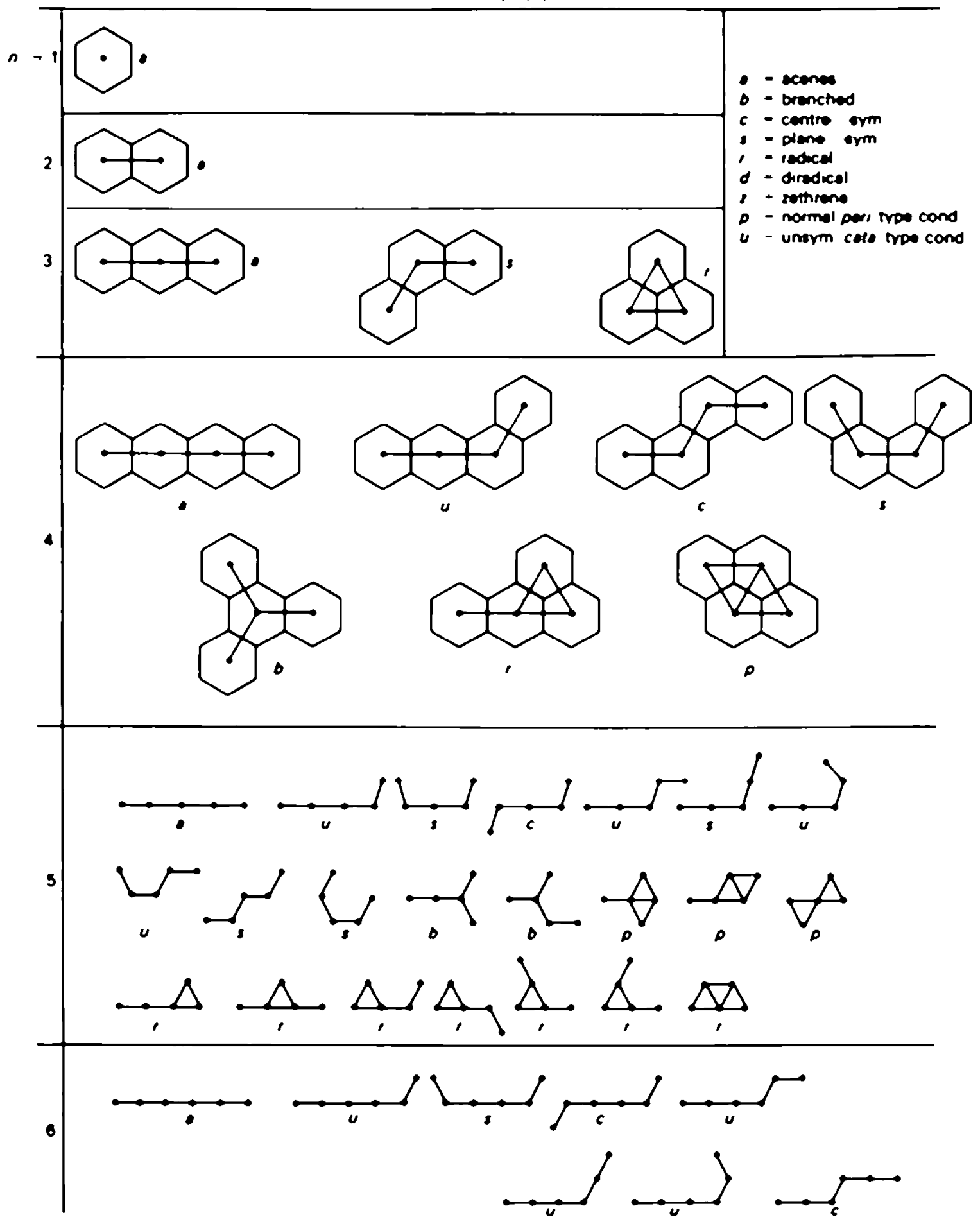




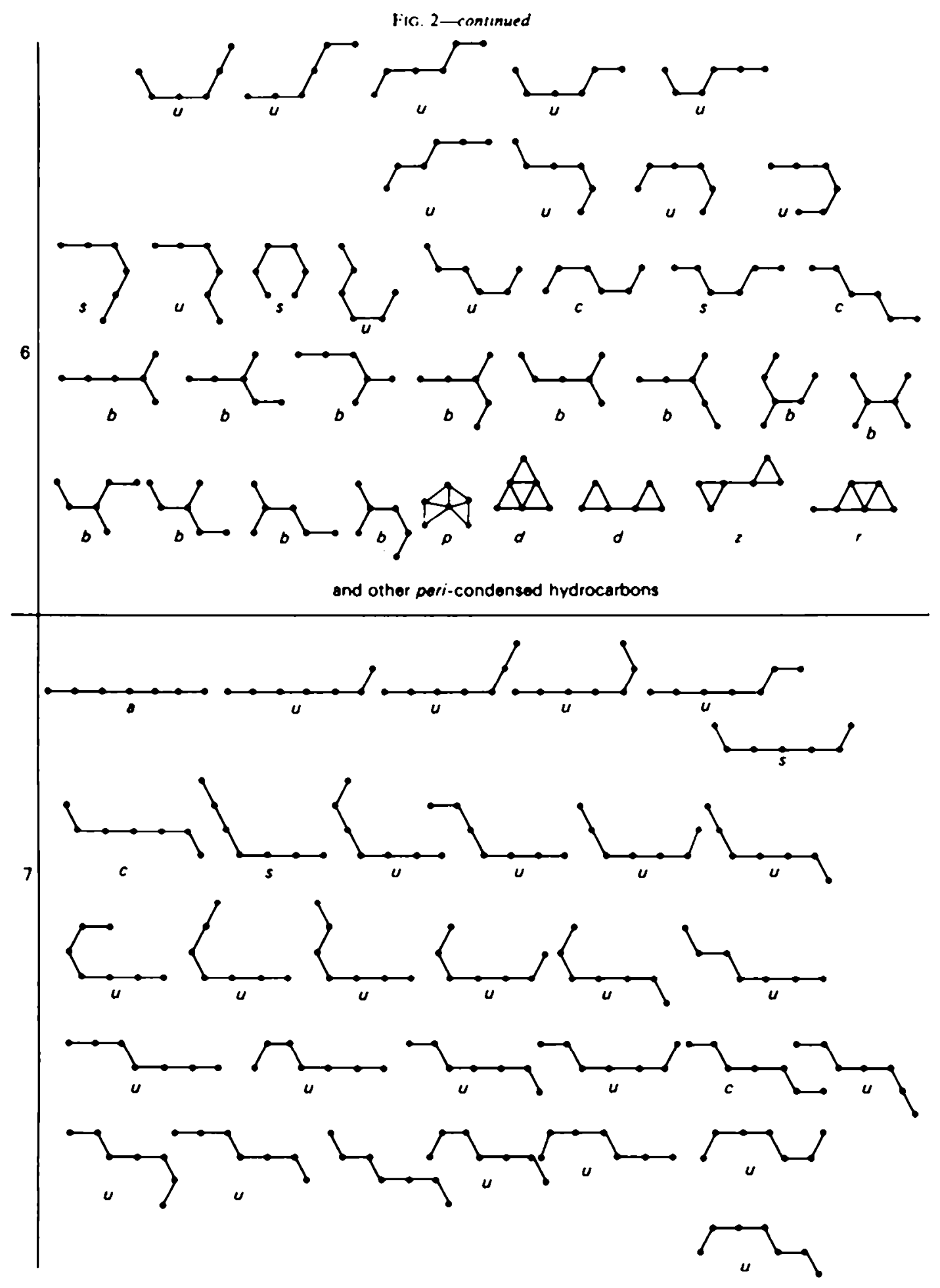




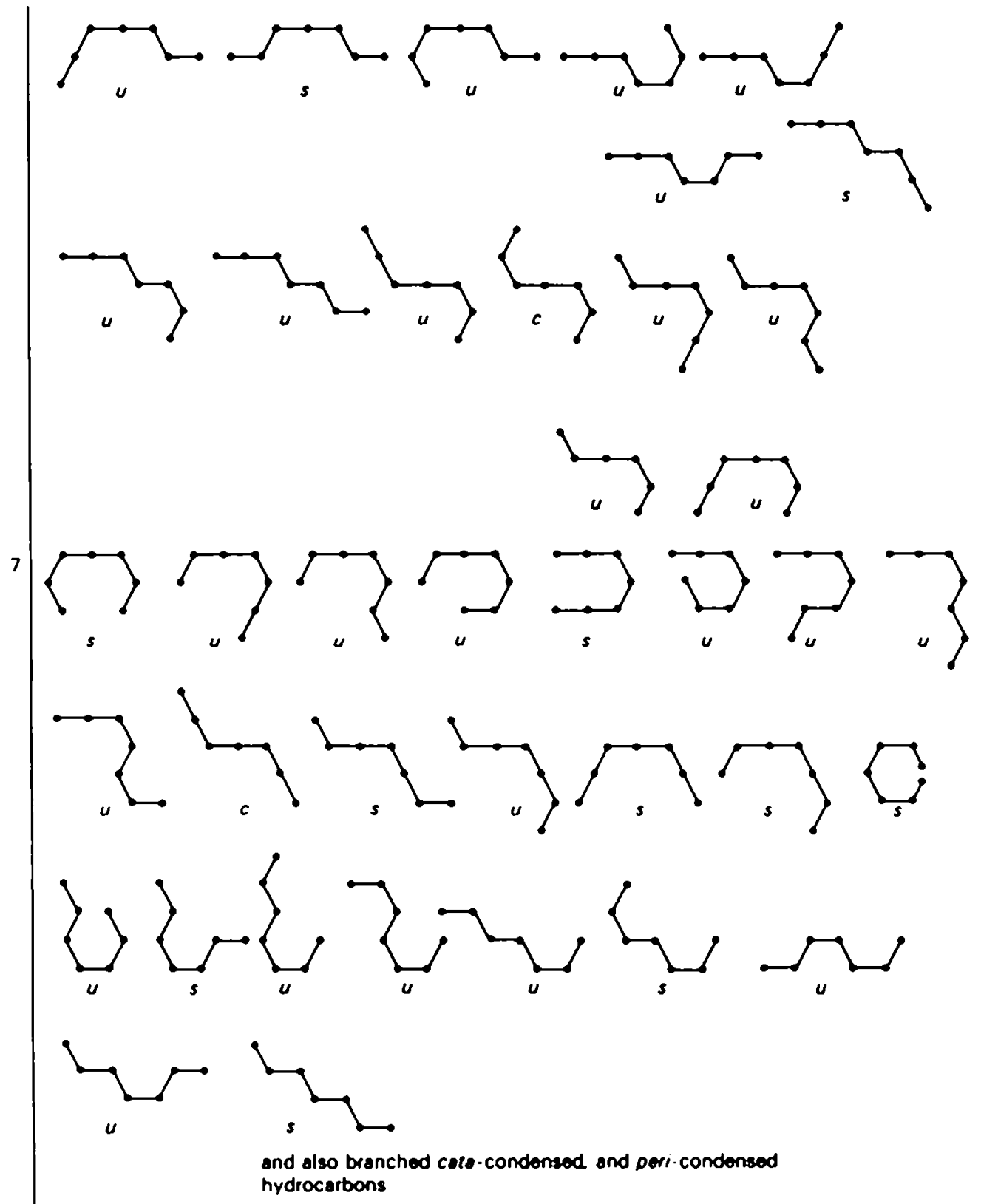


TABLF 1. EMPIRICAL NZMEERS OF ISOMERS (OH CONDE NSFD POT.YCYCZIC ARENAS

\begin{tabular}{|c|c|c|c|c|c|c|c|c|c|c|c|c|}
\hline \multirow{3}{*}{$\begin{array}{c}\text { No. of } \\
\text { hexagons }\end{array}$} & \multicolumn{6}{|c|}{ Cala-condensed } & \multicolumn{5}{|c|}{ Peri-condensed } & \multirow{3}{*}{$\begin{array}{l}\text { Grand } \\
\text { total }\end{array}$} \\
\hline & \multicolumn{5}{|c|}{ Non-branched } & \multirow{2}{*}{ Brancher } & & & & & \multirow{2}{*}{ Total } & \\
\hline & 1 & & & 4 & Total & & & & & & & \\
\hline$n$ & $a$ & s & $c$ & $u$ & $C_{n}$ & $b$ & $p$ & ? & $d$ & $z$ & $P$. & $c+b+P$ \\
\hline 1 & 1 & - & - & - & 1 & .. & - & -- & - & - & & 1 \\
\hline 2 & 1 & - & - & - & 1 & - & . & & & & .. & 1 \\
\hline 3 & 1 & 1 & . & & 2 & - & 1 & -- & - & - & 1 & 3 \\
\hline 4 & 1 & 1 & 1 & 1 & 4 & 1 & 1 & 1 & & & 2 & 7 \\
\hline 5 & 1 & 4 & 1 & 4 & 10 & 2 & 3 & 7 & - & & 10 & 22 \\
\hline 6 & 1 & 4 & 4 & 4 & 25 & 12 & 14 & 28 & 2 & 1 & 45 & 82 \\
\hline 7 & 1 & 13 & 4 & 52 & 70 & - & $\bullet$ & - & - & - & - & $333^{x .4}$ \\
\hline 8 & 1 & 13 & 13 & $169^{\circ}$ & $196^{\circ}$ & $\bullet$ & • & - & - & • & - & $1448^{\circ}$ \\
\hline
\end{tabular}

- Not determinod

- Calculated after Eqs (7b) and (8b).

- Results of computer calculations by L. S. Kassel (pers. comm.)

- Personal communication from Dr. Martin Gardner.

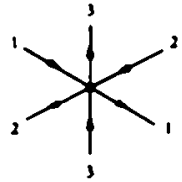

A<smiles>IC(I)(I)C(I)(I)I</smiles>

B

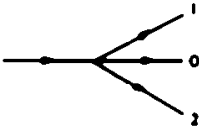

(

FIG. 4.

The present discussion is limited to non-branched, cata-condensed systems. Their associated skeleton (characteristic) trees differ from ordinary trees in that a kinked and a straight line are not equivalent (cf. II and III). The directions of the lines in the graph being the same as before (Fig. $4 A$ and $B$ ), the tree may be described by a number consisting of the digits $\pm 1, \pm 2, \pm 3$ with the restriction that only sequences $11,13,13,21,22,23,31,32,33$, and the same with reversed signs, are allowed. An alternative simpler notation of the tree makes use of the observation that two lines incident to the same point can make angles of $120^{\circ}, 180^{\circ}$ or $240^{\circ}$. Two adjacent lines with an angle of $180^{\circ}$ will be denoted by 0 , while two adjacent lines with angles $120^{\circ}$ or $240^{\circ}$ will be denoted by 1 or 2 (Fig. $4 \mathrm{C}$ ). The characteristic tree of a non-branched cata-condensed arene with $n$ hexagons (which is a directed path from one endpoint to the other) will therefore be denoted by a number of $n-2$ digits formed from digits 0,1 and 2. Examples of such numbers are: 1 or 2 (for II), 0 (for III), 11111 or 22222 (for IX).

One isomer may be indicated by one, two or four numbers, e.g. the above examples for II, III and IX, and $01,02,10$ or 20 for tetraphene. Acenes are the only compounds whose tree is a straight line, indicated by one number $00 \ldots 00$; in Table 1 and Fig. 2 they are indicated by $a$. Symmetrical trees, either relatively to an axis (they are noted by $s$ ) or to a center (noted by $c$ ), are described by two numbers which differ by 
interchanging 1 with 2 ; their endpoints are equivalent i.e. the same number is obtained if any of them is the surting point. Unsymmetrical ires (denoted by $u$ ) are described by four numbers diftering by the starting point or by interchenging digits 1 and 2 . The heading of Table 1 indicates for non-brancbed cala-ondensed arenes: I number for $a, 2$ for $s$ or $c$, and 4 for $u$. Since there are $3^{-2}$ numbers formed from $n-2$ dipits 0,1 or 2, which must indicate all $C$. isomers of a non-branched cala-condensed arene composed of $n$ heragons. one can write equation (2). Fqs 1 and 3 are straightfoward.

$$
\begin{aligned}
& c_{*}=a_{0}+s_{0}+c_{0}+u_{*}
\end{aligned}
$$

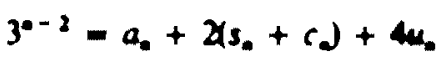

$$
\begin{aligned}
& \text { a. }-1 \\
& s_{2 n-1}=4\left(3^{-2}-1\right) \text { for } n \text { odd } n-2 m-1 \\
& \text { 32= } \left.-H^{3-1}-1\right) \text { for } n \text { even, } n-2 m \\
& c_{2 n},=y^{\left(3^{-1}-1\right)} \text { for } n \text { odd, } n=2 m-1 \\
& c_{2}-1\left(3^{-1}-1\right) \text { for } n \text { even. } n=2 m
\end{aligned}
$$

Equations (4) and (5) can be demonstrated by induction. Symmetrical trees (presented in Fig. Sh, $s_{-}$or $c_{\text {a }}$ are obuined from every symmetrical tree $s_{\infty}, 2$ and $c_{\infty}, 2$ respectively, by adding two lines at the endpoints at angles $120^{\circ}, 180^{\circ}$ or $240^{\circ}$ : one more tree is derived from the acene $a_{a}, 2$; therefore we have the recurrence formulas (6). which with Fig. 5 gives Eqs (4a) (Sb).

$$
s_{*}=3 s_{n}+1: \quad c_{n}=3 c_{n}+1
$$

From Eqs (1) H(Sb) we obtain:

$$
\begin{aligned}
& u_{2 m-1}=f\left(3^{2 m-3}-3^{m-1}-3^{2}+1\right) \quad \text { for } n \text { odd } n=2 m-1 \\
& u_{1 m}=k\left(3^{-1}-1\right)^{2} \quad \text { for } n \text { even, } n=2 n \\
& \left.C_{2 m-1}=13^{2 n}+3^{--1}+3^{-1}+1\right) \quad \text { for } n \text { odd, } n-2 n-1 \\
& C_{2 n}=\left(3^{-1}+1\right)^{2} \quad \text { for } n \text { even, } n=2 n
\end{aligned}
$$

The number $C_{\text {. }}$ of isomers calculated by means of formulas $(8 a)$ and (8b) gives results consistent with Table 1.

The nomenclature of non-branched caracoodensed arenes can be simply derived from the characteristic (skeleton) graph : the smallest number denoting the steleton. in brackets (this number has $n-2$ digits), is followed by the Greek numeral indicating the number $n$ of hexagons with the suffix catafusene. Some graph-systematic names are indicated in Table 2 . They are casily converted into a pictorial repreanution by drawing the tree ( 1 and 2 mean left or richt, 0 means straight on) and then completing the hexagonal frame. These names are of practical value for $n>4$.

In the future these ideas will be applied to branched cala-condensed arenes. and possibly also to peri-condensed syrtems. 


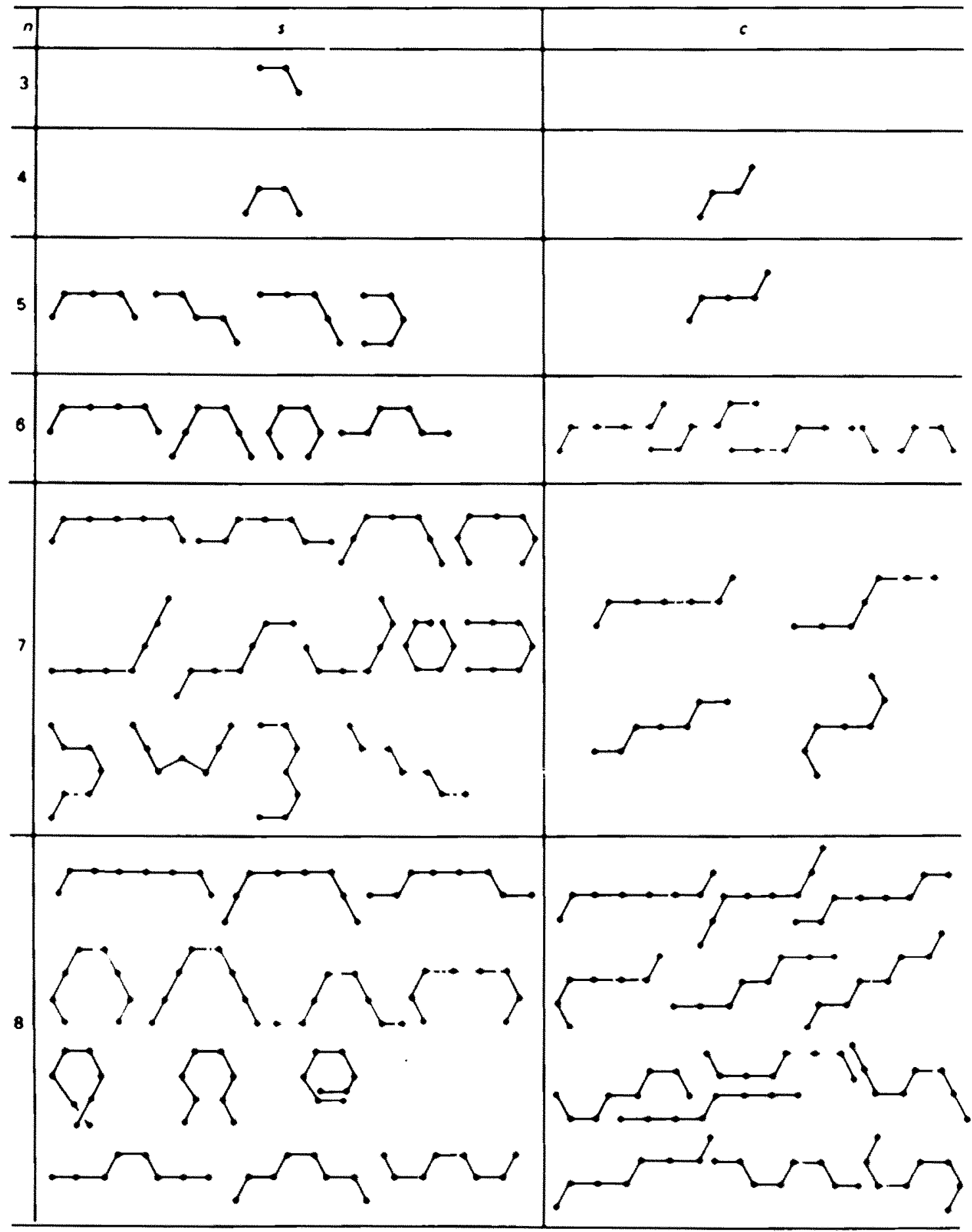

Hi S Symmetexai teen of types and $c$ 
TABLF 2. CORRESPONDENCZ RPT WEEN TRIVIAL. AND GRAPH-SYSTFMATIC

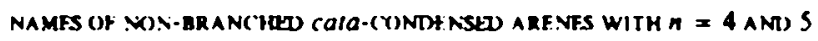

Tetracene

Tetraphene

3. 4-Benzophenanthrene

Chrysene

Pentacene

Picene

5.6-Benzochrysene

3:4.5 6. Dibenzophenanthrene

$1: 25: 6$-Dibenzanthracene

$1: 2,7: 8$-Dibenzanthracene

1 : 2-Benzotelraphene

3:4-Benzotetraphene

Pentaphene

1 : 2-Benzotetracene
(00) Tetracalafusene

(01) Tetracalafusene

(11) Tetracalafusene

(12) Tetracalafusene

(000) Pentacatafusene

(121) Pentacatafusenc

(112) Pentacatafusene

(111) Pentacatafusene

(102) Pentacalafusene

(101) Pentacatafusene

(011) Pentacatafusene

(012) Pentacatafusene

(010) Pentacatafusene

(001) Pentacatafusene

\section{REFERENCES}

' Procodung part in the seriea A.T. Balaban and F. Harary. Rev. Rummaine Chim in press.

2 Research supported in part by Grant GN-2544 from the National Scrence Foundation. USA. Chemical Information Program.

'E. Clar, Polycyclic Hydrocarbons. Academic Press-Springer Verlag. London (1964).

- IUPAC. Nomenclature of Organic Chemistry. 1957. Butterworths Scientific Publ.. London (1958): J. Am Chem Soc. 825545 (1960).

- The Naming and Indexing of Chemical Compounds from Chemical Abstracts. Introduction to the Subject Index. Chem Abstr. S6. Jan-June (1962)

- A. M. Patterson. L. T. Capell and D. F Walker, Ring Index (2nd Edition). Am. Chem. Soc., Washington $(1960)$.

I Loderberg. Proc. Natl. Acad. Sci. U.S.A. 53. 134 (1965): NASA Reports CR-57029. CR-68898. CR-68899

- B. Pullman. The Application of Waie Mechanical Meshads to the Study of Molecular Properives (Fdited by R. Daudelh Adr. in Chem. Phys. Vol. 8; p. 163. Interscience, London (1965).

G M. Bedger. The Chemical Basis of Carcinogenic Activity (Fdiled by I. N. Kugelmass) Ch. Thomas Publ. Springfield. Ill. Amer. Locture Series (1962):

H. Busch. An Introduction to the Biachemistry of the Cance Cell p. 207. Academic Press. New York (1962).

- A T. Balaban and C. D. Nenitzescu. Friedel Crafts and Related Reactions (Fdited by (;. A. Olah) Vol 2: p. 979 . Wiley-Interscience $(1964)$.

10 A. T. Balaban, Studii ji Cercetori Chem. Acad. R.S. Románia 13, 1299 (1965).

11.W. Jenny and R. Peler. Angew Chem. 77. 1027 (1965): Angew. Chem internat. Ed 4. 979 (1965):

- H. A. Staab and F. Binniz. Chem. Ber 100, 293 (1967).

12 M. Flammang-Barbieux. J. Nesielski and R. H Martin. Tetrahedron Letters 743 (1967).

13 F. Harary. Publ. Math. Inst. Hungar. Acad. Sci. 5.63 (1960).

14 F. Harary. Applied Combinatorial Mathematics (Editad by F. F Bockenbach) p. 185. Wiley. New York (1964).

19 F. Harary. Graph Theory and Theoretical Physics (Edited by F. Harary) Chapler 1. Section S. Academic Press, London (1967).

10 F. Harary. Ibid. Chapter 1. Section 6.

"C A. Coulson and H. C. Longuet-Higgins. Proc. Roy. Soc. A 19216 (1947).

1" E. Clar. Terratedron 3. 325 (1958): E Clar and A. McCallum. Ibid. 10. 171 (1960): E. Clar and I. A. McPherson. Ihid. 18. 144 (1962); E. Clar, Z. Chem. 2. 35 (1962); Liebigs Ann. Chem 669.44 (1963): Chimia 18. $375(1964)$. 
19 V. E. Sahini. J. Chim. Phys. 177 (1962): Rer. Chim. Acad. R.P. Roumaine 7, 1265 (1962): Ret. Chim. (Roumania) 15. 551 (1964).

20 M. Gordon and W. H. T. Devison. J. Chem Phys. 20.428 (1952).

2" M. Suunders, Tetrahedron 23, 2105 (1967). 